\title{
Una estrategia para la educación inclusiva
}

\section{A strategy for Inclusive Education}

\author{
Maestrante en Educación, Universidad Autónoma del Caribe. Coordinador Secretaría de Educación del Magdalena \\ jecheverriadelvalle@hotmail.com \\ Jorge Isaac Echeverría Del Valle \\ Maestrante en educación, Universidad Autónoma del Caribe. Docente, Secretaría de Educación del Magdalena \\ lourdesrua65@yahoo.es, \\ María De Lourdes Rúa Quintero \\ Maestrante en educación, Universidad Autónoma del Caribe. Psicosocial del plan retorno del Departamento de la Prosperidad Social, \\ Eleacidpertuz@hotmail.com, \\ Eleacid Isaac Pertuz Miranda
}

Recibido agosto 17 de 2012

Aceptado: sept.22 de 2012

\section{RESUMEN}

Teniendo en cuenta que la educación es un pilar fundamental en la formación integral del ser humano., se constituye en un deber de las instituciones educativas publicas promover la igualdad al garantizar el derecho a la educación, de todo niño, niña y joven, para acceder a un sistema donde se respeten y acepten las múltiples diferencias reconociendo la diversidad. Por lo anterior, se realizó un estudio, de tipo cuantitativo, utilizando estadísticas descriptivas propias de los índices de inclusión, que incluyeran categorías desde la perspectiva directiva, de academia, administrativa y comunidad: está alude a docentes, estudiantes y padres de familia. Con una muestra de cuatro (4) de las cinco (5) instituciones educativas públicas del municipio de Aracataca, Magdalena (Colombia). Para la recolección de la información se utilizó el instrumento diseñado por el Ministerio de Educación Nacional (MEN), que consta de dos formatos de encuesta una dirigida a docentes, directivos docentes y la otra a padres de familia, estudiantes y administrativos, lo que permitió calcular el índice de inclusión, en las instituciones educativas públicas del municipio en mención. Los resultados, fueron analizados con el enfoque de teóricos como la UNESCO, Lady Meléndez, quienes fueron un soporte para diseñar la estrategia de educación inclusiva, que tiene como finalidad la atención de la población estudiantil en condición de vulnerabilidad.

Palabras Clave. Diversidad, docente, escuela, estudiante, familia, índice de inclusión, población vulnerable

\begin{abstract}
Given that education is a fundamental pillar in the integral formation of the human being; so that it becomes a duty of public educational institutions to promote equality by ensuring the right to education to every child and youngsters to access a system where by recognizing diversity, the many differences are respected and accepted. Therefore, a study was conducted, quantitative, using descriptive statistics own inclusion rates, which included categories from the directive perspective, academia, administrative and community: is referring to teachers, students and parents. With a sample of four (4) of the five (5) public educational institutions of the municipality of Aracataca, Magdalena (Colombia)Therefore, we conducted a quantitative type study, under the analytical and empirical paradigm. To collect the information we used the instrument designed by the Ministry of National Education (MEN), which consists of two survey formats one addressed to teachers, head teachers and the other to parents, students and administrators, allowing calculate the rate of inclusion in public educational institutions of the municipality in question. The results were analyzed with the approach of theorists such as UNESCO, Lady Melendez, who were a support for designing the strategy of inclusive education, which aims to the attention of the student population in condition of vulnerability
\end{abstract}

Key words. Diversity, teacher, school, student, family, inclusion rate, vulnerable population. 


\section{Introducción}

Al pensar la escuela como un lugar para todos, se hace necesario diseñar estrategias colectivas que permitan concebir a cada ser humano como alguien único e irrepetible y con características particulares. Hay que tener en cuenta que el estudiante trae consigo historias individuales, que le hacen ver la vida de una manera diferente; lo anterior, no debe ser un impedimento para ser participe en los diferentes contextos educativos, sociales $y$ culturales.

La realidad para muchos niños, niñas y jóvenes en Colombia, esta empañada por situaciones como la discapacidad, el desplazamiento forzado, trabajo infantil, violencia, pobreza, drogadicción, prostitución, minorías étnicas o lingüísticas, y ubicación de viviendas sin acceso a escuelas cercanas. Todo lo anterior, lleva a que los docentes se enfrenten a múltiples realidades y necesidades intrínsecas dentro de su quehacer diario educativo; situación, que los obliga a replantear, su rol como actores participativos de la escuela e implementar acciones pedagógicas para atender de manera integral la diversidad de la población.

A los maestros que trabajan con menores con capacidades diferentes o características culturales diversas, se les presentan dificultades más relevantes; lo que no puede ser visto como un impedimento para transmitir una educación de calidad. Es un reto mayor, enseñar a toda una población con diversidad tan amplia, que cobije a estudiantes con discapacidad o sin ellas. Es importante tomar conciencia del papel que juegan todos los entes educativos públicos y la necesidad de implementar el programa de Educación Inclusiva, que incluya estrategias para brindar a este grupo de personas, las garantías de infraestructura, talento humano, material didáctico y proceso pedagógico, que propicien su vinculación y permanencia en el sistema educativo.

De acuerdo con lo manifestado por la UNESCO (2001), de los 140 millones de niños/as en el mundo, que se encuentran fuera del sistema educativo, la tercera parte de ellos presentan una discapacidad. Es necesario recordar que no es solo acceso en cuanto a cobertura, por lo cual la UNESCO realiza un seguimiento para la incorporación de esta población al sistema educativo.

Según Ramírez, Parrado, Londoño y Pereira (2010, p 17). “En Colombia doce (12) de cada cien (100) niños presentan una condición que limita su aprendizaje y participación, de los que solo tres asisten a la escuela".

Esta situación no solo impacta en la calidad de vida de las familias, sino que también incide negativamente en las perspectivas de crecimiento económico dentro de su comunidad lo que contribuye a perpetuar la desigualdad, pobreza e inequidad del país.

De acuerdo con las cifras arrojadas por el censo llevado a cabo por el DANE, en el año 2005, se destacan las siguientes cifras, tomadas como referencia.
- $\quad$ 1.059.000 niños y adolescentes trabajaban, con o sin pago, en la producción de bienes y servicios.

- La tasa de niñas entre 10 y 14 que han tenido hijos está en ascenso, 2.72 x 1.000 en 2006 y 2.80 en 2007, con una incidencia mayor en zonas rurales y pueblos. Estas cifras fueron refutadas por las Naciones Unidas ( 2005), en Colombia alrededor de 11.000 niños y niñas son víctimas de abuso sexual cada año.

- Según el Gobierno los desplazados a abril de 2008 eran 2.501.000, cifra que fue descalificada por Codhes (2010) quien considera que son más de 3,7 millones de colombianos los afectados por el desarraigo. 1.100.000 menores de 18 años han sido desplazados en los últimos 15 años, al tiempo que afirma que podría haber entre 6.000 y 11.000 niños y niñas reclutados por grupos armados al margen de la ley.

- En Colombia, más del 6\% de la población tiene alguna discapacidad (DANE, 2005). Todos ellos encuentran barreras que restringen su participación en la sociedad, que se refleja en los bajos niveles de escolaridad, la escasa formación técnica y profesional, la falta de oportunidades productivas y laborales, y las restricciones para el acceso a los servicios sociales. Esta situación convierte a la discapacidad en causa y efecto de la pobreza.

- En el departamento del Magdalena existen 63.243 personas con limitaciones, sin embargo se advierte que aún faltan 42.247 personas por registrar; de igual manera se resalta en el censo realizado por el DANE en el 2005.

El principio de Educación Inclusiva se aprobó en la Conferencia Mundial de Salamanca sobre las necesidades educativas especiales.

En ella se describe a la Educación Inclusiva como "un proceso para tomar en cuenta y responder a las diversas necesidades de todos los estudiantes por medio de prácticas inclusivas en aprendizaje, culturas y comunidades, y reduciendo la exclusión dentroy de la educación. Esto implica cambios y modificaciones, de contenido, enfoques, estructuras y estrategias con una visión común que cubre a todos los niños(as) del rango apropiado de edad y una convicción de que es la responsabilidad del sistema regular educar a todos los niños(as)... La Educación Inclusiva implica que todos los niños(as) y jóvenes con necesidades educativas especiales deberán de ser incluidos en los arreglos hechos para la mayoría de los niños(as)... Las escuelas inclusivas deben reconocer y responder a las diversas necesidades de los estudiantes, arreglos tanto en diferentes estilos como al ritmo del aprendizaje y asegurando la calidad de la educación para todos por medio de un currículo apropiado, dando lugar tanto a arreglos organizacionales, estrategias de enseñanza, uso de recursos y asociaciones con sus comunidades." (UNESCO, 1994. P.171) 
Según lo expresado anteriormente por la UNESCO (1994), se debe garantizar una educación integral de calidad para todos y todas, a la hora de tener acceso a la educación se pretende con ello superar las exclusiones y desigualdades que afectan a las personas, generando así pertinencia de los aprendizajes, una óptima formación individual, que redunde en el desarrollo social, económico y cultural para los pueblos.

Se pretende entonces que en el mundo las escuelas sean inclusivas; es decir, que atiendan tanto a pares sin discapacidad como a los estudiantes con cualquier deficiencia o con necesidades educativas especiales, para eso hay que adaptar las escuelas a todo este proceso, y es en este campo en el que se aborda este proyecto de investigación haciendo énfasis en el municipio de Aracataca, en donde el programa de educación inclusiva ha tenido ciertas falencias, actualmente se encuentra estancado y aquellos estudiantes en condición de vulnerabilidad están encontrando dificultades para ingresar y permanecer en las escuelas.

No es solo matricular a este tipo de población, sino garantizar su permanencia en el aula con los demás estudiantes; para eso la escuela debe adaptar el currículo de acuerdo a las necesidades y estilos de aprendizaje de cada uno, con el compromiso de los docentes, quienes al tiempo deben diseñar estrategias de enseñanza - aprendizaje, especificas, para estos estudiantes; así mismo se debe propender por mejores infraestructuras locativas tendientes a garantizar la permanencia de este tipo de población.

Por lo cual se realizó un análisis detallado de los factores que inciden en la garantía y permanencia de toda la población del municipio dentro de las instituciones educativas dando como resultado la investigación y publicación de este artículo; que toma como objeto de estudio la educación inclusiva, a su vez el campo de acción lo constituyen cuatro de las cinco instituciones educativas departamentales (John F. Kennedy, Elvia Vizcaino de Todaro, Fossy Marcos María, Buenos Aires) de donde se seleccionó la muestra ya que una de las instituciones (Institución Educativa Departamental Gabriel García Marquez) no se acogió a participar en el programa de educación inclusiva propuesto. Dando como resultado la formulación del siguiente problema científico ¿Cómo lograr una Educación Inclusiva en las escuelas públicas del municipio de Aracataca?

Donde se propuso como objetivo diseñar una estrategia de educación inclusiva para las escuelas públicas del municipio de Aracataca. Magdalena (Colombia).

Para alcanzar el objetivo propuesto, se acometieron las siguientes tareas: Se revisaron los fundamentos teóricos y legales que sustentan la educación inclusiva; se diagnosticaron las condiciones para desarrollar la educación inclusiva en las escuelas públicas del municipio de Aracataca. Con los resultados, se diseñó una estrategia para la educación inclusiva teniendo en cuenta el sector educativo público en el contexto del municipio de Aracataca, Magdalena.

\section{Metodología}

Esta investigación fue de tipo cuantitativo, bajo el paradigma empírico analítico, de carácter descriptivo, para lo cual se utilizó el instrumento diseñado por el Ministerio de Educación Nacional (MEN) que permite calcular el índice de inclusión.

Para el desarrollo de esta investigación la población objeto de estudio la constituyen padres de familia, estudiantes, docentes, directivos docentes y administrativos vinculados a las instituciones educativas públicas del municipio de Aracataca- Magdalena, como se muestra en la siguiente tabla:
$\mathrm{N}=5$ Instituciones educativas públicas del municipio de Ara- cataca- Magdalena
Se trabajó con cuatro porque una no se acogió al programa de educación
Inclusiva.

La selección de la muestra para la aplicación de los cuestionarios del Índice de Inclusión se estableció siguiendo el criterio estadístico del nivel de confiabilidad del 95\%, implementándolo para grupos de docentes, estudiantes, padres de familia y acudientes; en el caso de directivos docentes, personal administrativo y de apoyo.

Tabla 1. Población de docentes, directivos docentes, administrativos, estudiantes y padres de familia de las Instituciones Educativas del municipio de Aracataca, Magdalena.

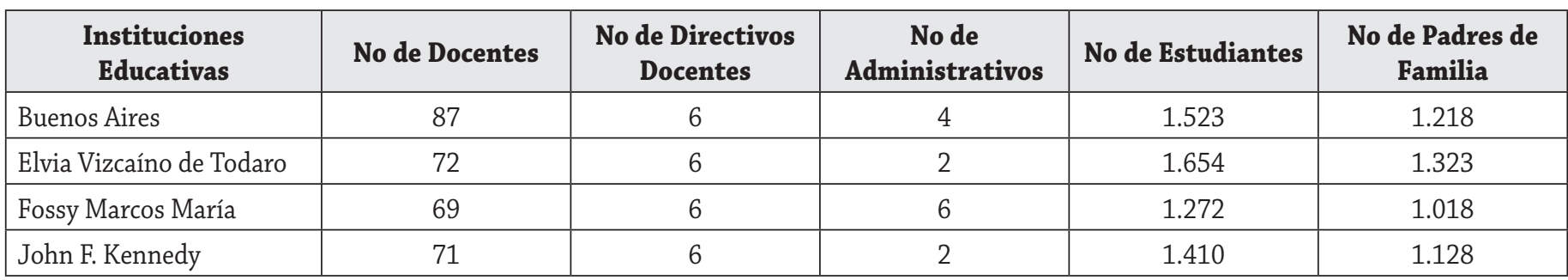

Fuente: Dirección de Núcleo de Desarrollo Educativo No. 01 del Departamento del Magdalena. 
En poblaciones relativamente pequeñas (menores de 50) se recomienda cubrir la totalidad de la población, para que no se subestime el Índice al obtener los resultados en las diferentes áreas y procesos de gestión directiva, académica, administrativa y de la comunidad. En la siguiente tabla se define la muestra a encuestar

Para el diseño de la estrategia de educación inclusiva en las escuelas públicas del municipio de Aracataca, los investigadores se apoyaron en el índice de inclusión como una herramienta de autoevaluación que permite conocer las condiciones de inclusión de las instituciones educativas objeto de estudio. Para obtener el índice de inclusión se utilizó un cuestionario estructurado desde las cuatro áreas de gestión: directiva, administrativa, académica, y comunidad. El índice presenta dos tipos de cuestionarios: uno para docentes, directivos docentes, personal de apoyo y administrativo; otro para padres de familia y estudiantes, los cuales se anexan.

En la implementación de las dos encuestas, se hizo necesaria la realización de un cronograma de actividades:

- Reunión con los rectores de las diferentes instituciones educativas públicas, donde se socializo el proyecto de investigación.

- Charla sostenida con los docentes, directivos docentes, administrativos para socializar el proyecto y posteriormente se aplicó el cuestionario número 1.

- Se siguió, el proceso de socialización con los padres de familia y estudiantes de las instituciones objeto de estudio, procediendo a aplicar el formulario número 2.

- Una vez recopilada la información contenida en los formularios se procede a procesar en el software diseñado por el MEN obteniéndose el diagnostico de cada institución y el índice de inclusión municipal, cuyos resultados se muestran a continuación.

\section{Resultados}

Cabe señalar, que los resultados obtenidos están sustentados en el Índice de inclusión, el cual es una herramienta que permite a la institución educativa realizar el proceso de autoevaluación de la gestión inclusiva, reconociendo el estado actual en la atención a la diversidad, el análisis de las fortalezas y oportunidades de mejoramiento para establecer prioridades y tomar decisiones que cualifiquen las condiciones de aprendizaje, participación y convivencia de la comunidad.

Una vez aplicado el cuestionario para obtener el Índice de Inclusión de la institución educativa, se procedió, a su tabulación, procesamiento de datos, análisis e interpretación, preparación de informe y se finalizó con la articulación de sus resultados con relación a los obtenidos en la guía de Autoevaluación No 32, expedida por el Ministerio de Educación Nacional. El proceso, se llevó a cabo con los siguientes pasos:

Paso uno. Tabulación: los datos arrojados en los cuestionarios aplicados a familias, estudiantes, docentes y directivos docentes se registran en la base de datos, en el programa en Excel.

\section{Paso dos. Análisis de los resultados del Índice de Inclusión:} el proceso de análisis tiene como finalidad obtener a partir de los datos estadísticos las tendencias de orden cuantitativo para cada estamento, en cada proceso, área de gestión y en el Índice global de la institución educativa.

Paso tres. Interpretación: se presentan los cuadros por rangos de puntajes para favorecer la interpretación de los resultados en cada proceso, área de gestión y el Índice global de la institución.

\section{PRESENTACIÓN E INTERPRETACIÓN PARA EL ANALISIS DE RESULTADOS}

A continuación se presentan los cuadros por rangos de puntajes que fueron tenidos en cuenta para favorecer la interpretación de los resultados en cada proceso, área de gestión y el Índice global de la institución, para que los autores apoyados en los mismos desarrollaran en el manual reportes de índices de inclusión, mediante una matriz en Excel. MEN, 2008.

Tabla 2. Muestra de docentes, directivos docentes, administrativos, estudiantes y padres de familia de las Instituciones Educativas del municipio de Aracataca, Magdalena.

\begin{tabular}{|l|c|c|c|c|c|}
\hline Instituciones Educativas & No. de Docentes & $\begin{array}{c}\text { No. de Directivos } \\
\text { Docentes }\end{array}$ & $\begin{array}{c}\text { No. de } \\
\text { Administrativos }\end{array}$ & $\begin{array}{c}\text { No. de } \\
\text { Estudiantes }\end{array}$ & $\begin{array}{c}\text { No. de Padres } \\
\text { de Familia }\end{array}$ \\
\hline Buenos Aires & 55 & 6 & 4 & 153 & 153 \\
\hline Elvia Vizcaíno de Todaro & 55 & 6 & 2 & 153 & 153 \\
\hline Fossy Marcos María & 55 & 6 & 6 & 153 & 153 \\
\hline John F. Kennedy & 55 & 6 & 2 & 153 \\
\hline
\end{tabular}

Fuente: Calculado por los autores tomando en cuenta la Guía de Educación Inclusiva. 
Cuadro No 1. Guía para la interpretación de resultados en los procesos y áreas de gestión.

\begin{tabular}{|c|l|}
\hline RANGOS & \multicolumn{1}{c|}{ BASES DE INTERPRETACIÓN } \\
\hline $\mathbf{0 . 0}$ - 0.99 & $\begin{array}{l}\text { Los resultados del Índice en este rango indican que no se desarrollan acciones inclusivas para la atención a la diversidad en el } \\
\text { proceso o área de gestión. }\end{array}$ \\
\hline $\mathbf{- 1 . 9 9}$ & $\begin{array}{l}\text { Los resultados del Índice en este rango indican que la comunidad educativa desconoce las acciones inclusivas que desarrolla la } \\
\text { institución para la atención a la diversidad, en el proceso o área de gestión. }\end{array}$ \\
\hline $\mathbf{2 . 0}$ - 2.79 & $\begin{array}{l}\text { Los resultados del Índice en este rango indican que en algunas ocasiones se realizan acciones inclusivas para la atención a la } \\
\text { diversidad en el proceso o área de gestión propia y son conocidas por la mayoría de los integrantes de la comunidad educativa. }\end{array}$ \\
\hline $\mathbf{2 . 8}$ - 3.49 & $\begin{array}{l}\text { Los resultados del Índice en este rango indican que con frecuencia se realizan acciones inclusivas para la atención a la diversidad } \\
\text { en el proceso o área de gestión y son conocidas por todos los integrantes de la comunidad educativa. }\end{array}$ \\
\hline $\mathbf{3 . 5}$ - 4.0 & $\begin{array}{l}\text { Los resultados del Índice en este rango indican una evaluación permanente para conocer el impacto de las acciones inclusivas } \\
\text { para la atención a la diversidad, en el proceso o área de gestión y la usa para aportar al desarrollo institucional. }\end{array}$ \\
\hline
\end{tabular}

Fuente: Guía de Educación Inclusiva Ministerio de Educación Nacional

Cuadro No 2. Guía para la interpretación de resultados, índice global.

\begin{tabular}{|c|l|}
\hline RANGOS & \multicolumn{1}{c|}{ BASE DE INTERPRETACIÓN } \\
\hline $\mathbf{0}$ - 0.99 & $\begin{array}{l}\text { Los resultados del Índice en este rango indican que en la institución no se formulan políticas ni se desarrollan acciones que } \\
\text { favorezcan las prácticas inclusivas para la atención a la diversidad. }\end{array}$ \\
\hline $\mathbf{1 ~ - 1 . 9 9}$ & $\begin{array}{l}\text { Los resultados del Índice en este rango significan que se formulan políticas y se realizan algunas acciones inclusivas de manera } \\
\text { desarticulada de la gestión institucional para la atención a la diversidad y son desconocidas por la mayoría de los integrantes } \\
\text { de la comunidad educativa. }\end{array}$ \\
\hline $\mathbf{2 . 0}$ - 2.79 & $\begin{array}{l}\text { Los resultados del Î́ndice en este rango indican que se realizan acciones organizadas en las áreas de gestión, conocidas por la } \\
\text { mayoría de los integrantes de la comunidad educativa; además se incluyen en los planes de mejoramiento las prioridades de } \\
\text { transformación institucional para cualificar la atención a la diversidad. }\end{array}$ \\
\hline $\mathbf{2 . 8}$ - 3.49 & $\begin{array}{l}\text { El resultado del Índice en este rango significa que las acciones inclusivas para la atención a la diversidad, formuladas en el plan } \\
\text { de mejoramiento, se desarrollan y evalúan de manera continua y están articuladas con la gestión institucional, lo que favorece } \\
\text { el aprendizaje, la participación y la convivencia de todos los integrantes de la comunidad educativa. }\end{array}$ \\
\hline $\mathbf{3 . 5 - 4 . 0}$ & $\begin{array}{l}\text { El resultado del Índice en este rango evidencia una cultura institucional caracterizada por buenas prácticas inclusivas, el trabajo } \\
\text { colaborativo, la pertenencia a redes de apoyo y por ser centro de referencia para la atención a la diversidad; además el plan de } \\
\text { desarrollo institucional está articulado con el de la entidad territorial. }\end{array}$ \\
\hline
\end{tabular}

Fuente: Guía de Educación Inclusiva del Ministerio de Educación Nacional, 2008.

ANÁLISIS DE RESULTADOS POR INSTITUCIÓN EDUCATIVA PÚBLICA EN EL MUNICIPIO DE ARACATACA, MAGDALENA

Con base a la Guía de Inclusiva del Ministerio de Educación Nacional, 2008 y su base de interpretación, se desarrollaron los índices de inclusión para cada institución pública en el municipio de Aracataca, Magdalena, los cuales se analizan a continuación:

Al analizar los datos en la aplicación del índice de inclusion en la Institucion Educativa Buenos Aires se obtuvieron los siguientes resultados:

El índice institucional fue de 2,91 donde se evidencio que las areas de mas bajo desempeño fue la comunidad $(2,66)$ la administrativa $(2,80)$ al tiempo que las de mayor desempeño es la directiva $(3,09)$ al igual que la academica con $(3,09)$. Al ubicar el índice de inclusion en este rango permite considerar que las acciones inclusivas para la atencion a la diversidad, formuladas en el plan de mejoramiento se desarrollan y evaluan de manera continua, estando articuladas con la gestion institucional, lo que favorece el aprendizaje, la participacion y la convivencia de todos los integrantes de la comunidad educativa.

Al realizar el análisis de los datos obtenidos en la aplicación del índice de inclusión en la Institución Educativa Elvia Vizcaino de Todaro se llegó a las siguientes conclusiones:

El índice institucional fue de $(2,25)$ evidenciándose las áreas de más bajo desempeño la administrativa con $(1,89)$ comunidad con $(2,33)$, al tiempo que se muestran las áreas de mayor desempeño fueron la académica con un puntaje de $(2,35)$ y la directiva con $(2,52)$. Al ubicar el índice de inclusión en este rango se muestra 
que en la institución se realizan acciones organizadas en las áreas de gestión, conocidas por la mayoría de los integrantes de la comunidad educativa; además se incluyen en los planes de mejoramiento las prioridades de transformación institucional para cualificar la atención a la diversidad.

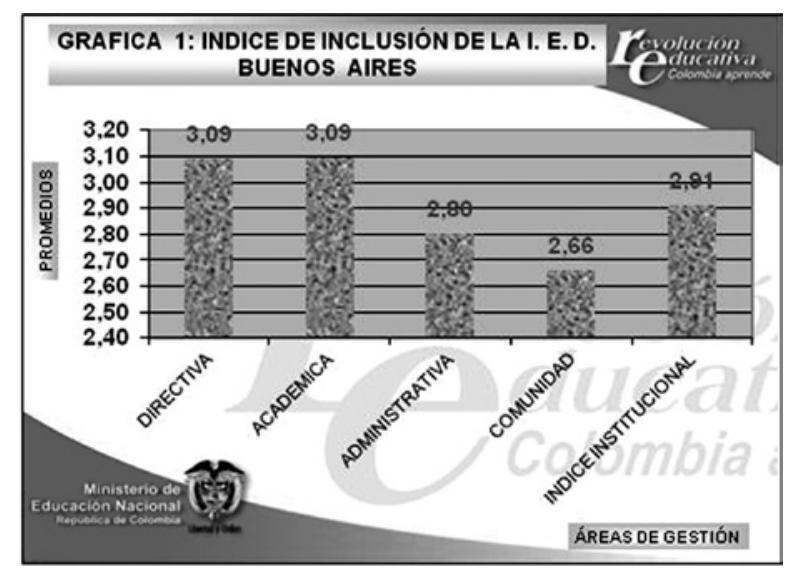

Fuente: Los autores apoyados en el manual para reportes de índices de inclusión, mediante una matriz en Excel. MEN, 2008.

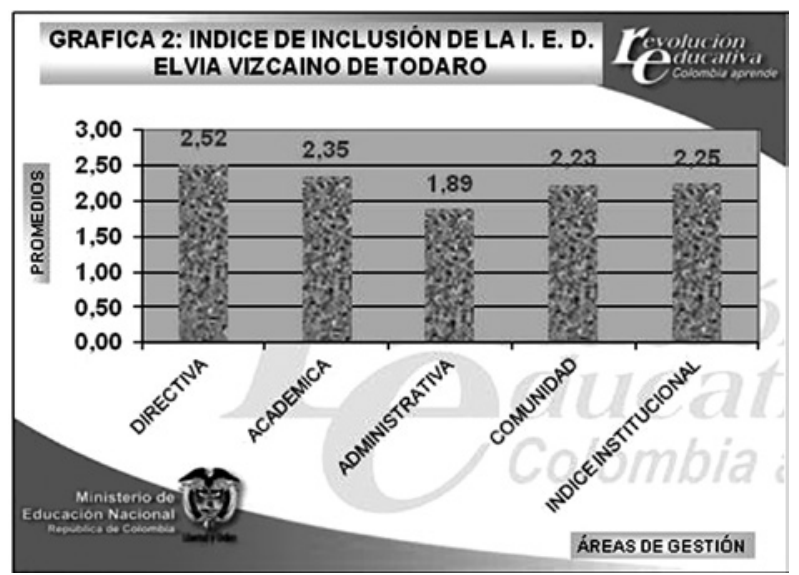

Fuente: Los autores apoyados en el manual para reportes de índices de inclusión, mediante una matriz en Excel. MEN, 2008.

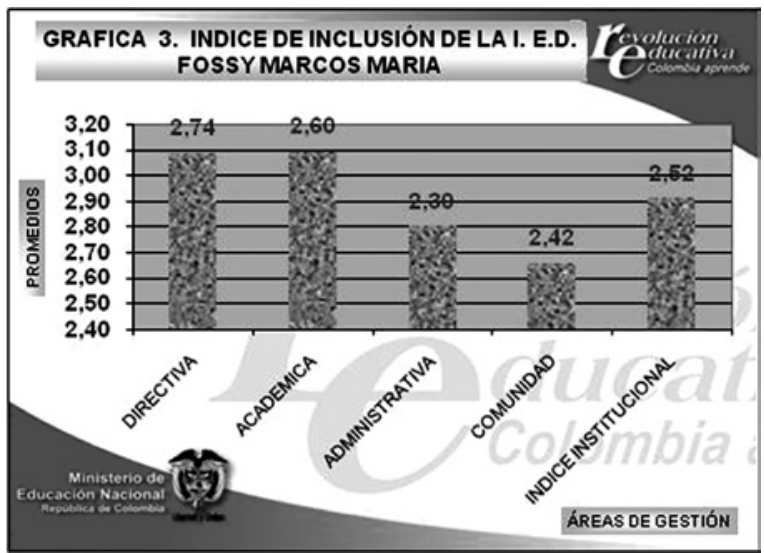

Fuente: Los autores apoyados en el manual para reportes de índices de inclusión, mediante una matriz en Excel. MEN, 2008.
Al realizar el analisis de los datos obtenidos en la aplicación del índice de inclusión en la institucion educativa Fossy Marcos Maria se llego a las siguientes conclusiones:

El índice institucional fue de (2,52) mostrando las áreas de mas bajo desempeño la administrativa con $(2,30)$, la comunidad $(2,42)$ y las de mayor desempeño la academica $(2,60)$ directiva $(2,74)$. Al ubicar el índice de inclusion en este rango se evidencia que en la institucion se realizan acciones organizadas en las areas de gestion, conocidas por la mayoria de los integrantes de la comunidad educativa; ademas se incluyen en los planes de mejoramiento las prioridades de transformacion institucional para cualificar la atencion a la diversidad.

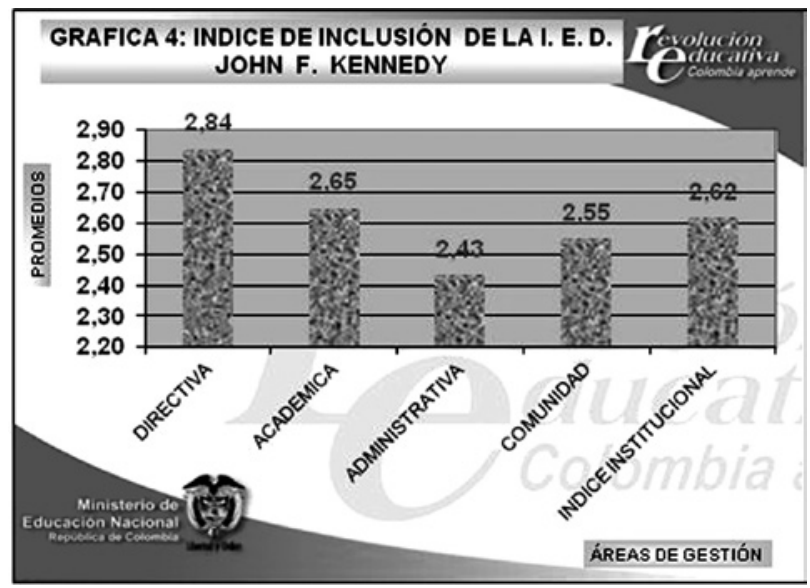

Fuente: Los autores apoyados en el manual para reportes de índices de inclusión, mediante una matriz en Excel. MEN, 2008.

Al analizar los datos obtenidos en la aplicación del índice de inclusión en la institución educativa John F. Kennedy se obtuvieron los siguientes resultados:

El índice institucional fue de $(2,62)$ donde se muestran las áreas de más bajo desempeño la académica con $(2,43)$ seguida por la comunidad con $(2,55)$ y las de mayor desempeño la académica con un puntaje de $(2,65)$ y la directiva con $(2,84)$. Al ubicar el índice de inclusión en este rango indica que se realizan acciones organizadas en las áreas de gestión, conocidas por la mayoría de los integrantes de la comunidad educativa; además se incluyen en los planes de mejoramiento las prioridades de transformación institucional para cualificar la atención a la diversidad.

En referencia al índice global del municipio de Aracataca, Magdalena, se puede decir que obtuvo un puntaje de 2,58, lo que indica que en las instituciones educativas del municipio objeto de estudio, se realizan acciones organizadas en las diferentes áreas de gestión, conocidas por la mayoría de los integrantes de la comunidad educativa, además se incluyen en los planes de mejoramiento las prioridades de transformación municipal para cualificar la atención a la diversidad. El área que presenta un resultado bajo es la gestión administrativa $(2,35)$ y la que más se destaca es el área de gestión directiva $(2,81)$. 


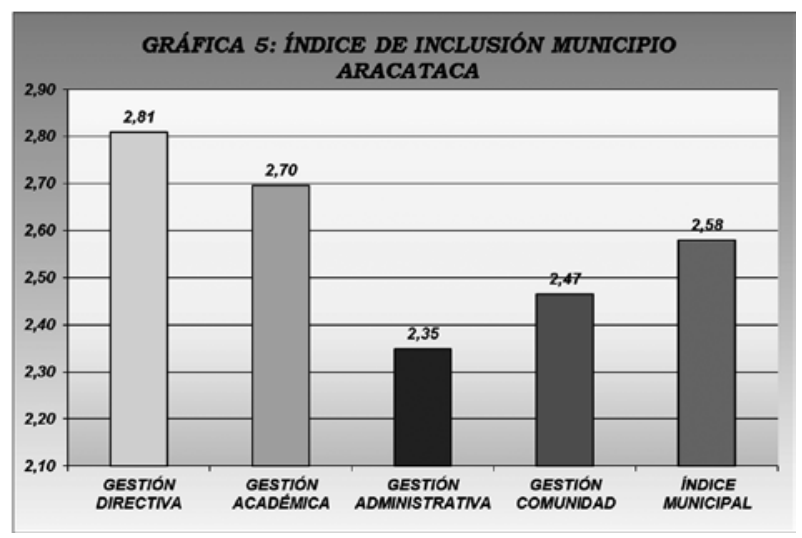

Fuente: Los Autores

\section{ANÁLISIS DE RESULTADOS COMPARATIVOS}

Al hacer un análisis de resultados de los índices de inclusión de las instituciones educativas del municipio de Aracataca - Magdalena, se puede concluir.

Elárea de gestión con menor desempeño para el índice de inclusión municipal fue la administrativa, con un puntaje de 2,35; de igual manera el área de gestión de la comunidad arrojó una puntuación de 2,47 , valores que están por debajo del índice municipal que es del 2,58; se hace necesario resaltar que las áreas de mayor desempeño fueron la gestión académica con un puntaje de 2,70 y la directiva con 2,81, lo que indica que se realizan algunas acciones inclusivas para la atención a la diversidad, que son tenidas en cuenta en los planes de mejoramiento institucional, pero no son evaluadas, lo que no permite una mayor cualificación para la atención a la diversidad.

\section{Conclusiones}

El comportamiento de los índices de inclusión de las instituciones educativas del municipio de Aracataca presentan una homogeneidad, debido a que en todas ellas se realizan acciones inclusivas que se reflejan en los Planes de Mejoramiento, pero que al no ser evaluadas, no permiten cualificar la atención a la población vulnerable ni la efectividad de las mismas, aunque se cumpla con el mero requisito de indicadores de inclusión y de la gestión desde lo administrativo, lo directivo, lo académico y lo comunitario.

Es por todo lo anterior que se hace necesario diseñar estrategias educativas inclusivas que permitan cualificar la atención y el seguimiento con indicadores de cómo son los resultados de esta población vulnerable en el Municipio de Aracataca, Magdalena.

\section{Referencias}

Ainscow, M. Desarrollo de escuelas inclusivas: ideas, propuestas y experiencias para mejorar las instituciones escolares. España. Narcea, 2001.

Balestrini, M. Como se elabora un proyecto de investigación. Editorial: Venezuela, 1997.

Blanco, R. (s.f) Hacia una escuela para todas y con todos. Recuperado de http://www.inclusionducativa.cl.

Booth, T. \& Ainscow, M. Guía para la evaluación y la mejora de la educación inclusiva, CSIE, Consorcio universitario para la educación inclusiva, Universidad Autónoma de Madrid, 2001

Calle L. \& Pórtela L. (2006). El concepto de la inclusión en la pedagogía.

Cerda, H. (1997) Como elaborar proyectos, diseño, ejecución y evaluación de proyectos sociales y educativos, editorial magisterio, Bogotá.

Departamento Administrativo Nacional de Estadísticas (DANE). Censo 2005.

Giné, C. (2003). Inclusión y sistema educativo. III Congreso "La Atención a la Diversidad en el Sistema Educativo". Universidad de Salamanca. Instituto Universitario de Integración a la Comunidad (INICO).

Grau, C. (1998). Educación especial: De la integración escolar a la escuela inclusiva. España. Promolibro.

Tabla 3. Comparativo instituciones educativas e índice de inclusión del municipio de Aracataca - Magdalena

\begin{tabular}{|c|c|c|c|c|c|}
\hline \multirow{2}{*}{$\begin{array}{c}\text { NOMBRE DE LA } \\
\text { INSTITUCIÓN EDUCATIVA }\end{array}$} & \multicolumn{4}{|c|}{ AREA DE GESTIÓN } & INDICE \\
\cline { 2 - 6 } & DIRECTIVA & ACADÉMICA & ADMINISTRATIVA & COMUNIDAD & INSTITUCIONAL \\
\hline Buenos Aires & 3,09 & 3,09 & 2,80 & 2,66 & 2,91 \\
\hline Elvia Vizcaino de Todaro & 2,52 & 2,35 & 1,89 & 2,23 & 2,25 \\
\hline Fossy Marcos Marí & 2,74 & 2,60 & 2,30 & 2,42 & 2,52 \\
\hline John F. Kennedy & 2,84 & 2,65 & 2,43 & 2,55 & 2,62 \\
\hline $\begin{array}{c}\text { İNDICE DE INCLUSIÓN } \\
\text { MUNICIPAL }\end{array}$ & $\mathbf{2 , 8 1}$ & $\mathbf{2 , 7 0}$ & $\mathbf{2 , 3 5}$ & 2,47 & 2,58 \\
\hline
\end{tabular}

Fuente: Los autores 
Hernández Sampieri, R. (1997). Metodología de la investigación tercera edición. Mc Graw Hill Interamericana.

Meléndez, L. (2002). La inclusión escolar del alumno con discapacidad intelectual, Glarp IIPD. Bogotá.

Meléndez, L. (2003). Atención a la diversidad en el contexto educativo costarricense. División de Desarrollo Curricular / MEP. (Mimeo).

Ministerio de Educación Nacional. (2008). Cartilla de educación inclusiva, guía No 34.

Ministerio de Educación de Colombia. Programa de Educación Inclusiva con Calidad. Convenio MEN - Tecnológico de Antioquia, Institución Universitaria. 2006-2007

Organización de las Naciones Unidas para la educación (UNESCO). (2004). Temario abierto sobre educación inclusiva, OREALC/ UNESCO, Santiago, Chile.

Ortega C. (2000) Ciencias políticas, democráticas y constitución $9^{\circ}, 10^{\circ}$ y $11^{\circ}$ de Bachillerato, Talleres de lito esfera Ltda, quinta edición, Santa Fé de Bogotá.

Ramirez, J. Parrado, C. Londoño, S. \& Villalba, B. (2010). Camino hacia una educación inclusiva. Santa Marta.

Suarez, P. (2009). Metodología de la investigación, Orión editores, Bogotá D.C.
UNESCO. (1994). Informe Final Conferencia Mundial sobre necesidades Educativas Especiales. Acceso y Calidad. Marco de Acción y Declaración de Salamanca. París: UNESCO/MEC.

UNESCO. (2000). Conferencia Mundial de Educación para Todos, Evaluación diez años después del Foro Mundial sobre la Educación (Jomtien-Tailandia),

UNESCO. (2001). Nuevo compromiso mundial para la educación básica. En Marco Acción de Dakar. Educación para Todos: Cumplir nuestros compromisos comunes. MEP/SIMED.

UNESCO. (2004). Educar en la diversidad. Santiago de Chile.

UNESCO. (2004) Documento Temario abierto sobre educación inclusiva. Santiago de Chile.

Universidad de Antioquia. (2009). Programa de Educación Especial. Colombia

Vasco, C. (s.f) Tres estilos de trabajo en las ciencias sociales. Editorial CINEP, Bogotá.

Villanueva, Á. (2004). Ley general de educación articulación con la ley 715 - Reglamentación, concordancia, jurisprudencia y comentarios. Cooperativa editorial magisterio, Santa Fé de Bogotá. 